\title{
Diffuse brainstem glioma: prognostic factors
}

\author{
Clinical article \\ Marcos Dellaretti, M.D., ${ }^{1-3}$ Nicolas Reyns, M.D., Ph.D., ${ }^{1}$ Gustavo Touzet, M.D., ${ }^{1}$
François Dubois, M.D., ${ }^{1}$ Sebastião Gusmão, M.D., ${ }^{2}$
JÚlio LeOnardo Barbosa Pereira, M.D., ${ }^{2}$ And Serge Blond, M.D. ${ }^{1}$
}

${ }^{1}$ Department of Neurosurgery, CHRU Hôpital Roger Salengro, Lille, France; ${ }^{2}$ Department of Neurosurgery, Santa Casa Hospital; and ${ }^{3}$ Faculty of Medicine, Federal University of Minas Gerais, Belo Horizonte, Brazil

\begin{abstract}
Object. Brainstem gliomas were regarded as a single entity prior to the advent of MRI; however, several studies investigating MRI have recognized that these lesions are a heterogeneous group, and certain subgroups have a better prognosis for long-term survival. The aim of this study was to conduct a retrospective analysis of prognostic factors of patients with brainstem gliomas confirmed by histopathological diagnosis, particularly regarding assessment of whether histological grade, age, and MRI findings are prognostic factors for patient survival.

Methods. The study evaluated 100 patients diagnosed with brainstem glioma. There were 63 adults (40 men and 23 women; age range 18-75 years, mean 41 years) and 37 children (19 boys and 18 girls; age range 2-12 years, mean 6.9 years).

Results. The mean overall survival of this population, measured from the date of biopsy, was 57 months for diffuse low-grade glioma and 13.8 months for diffuse high-grade glioma $(\mathrm{p}<0.001)$. The mean survival among patients with nonenhancing contrast lesions on MRI was 54.2 months, whereas for patients with enhancing lesions, it was 21.7 months $(\mathrm{p}<0.001)$. Comparisons between the Kaplan-Meier survival curves of adults and children revealed similar median survival periods of 25 and 16 months, respectively ( $p>0.05)$. The multivariate analysis $($ Cox proportional hazards regression) revealed that only histological grade was a significant prognostic factor $(\mathrm{p}<0.001)$.

Conclusions. The study revealed that histological grade and MRI features were significant prognostic factors for survival in these patients, but in multivariate analysis, only histological grade remained a significant factor. (http://thejns.org/doi/abs/10.3171/2012.7.JNS111992)
\end{abstract}

\section{KeY Words • brainstem tumor diagnostic and operative techniques}

$\mathrm{P}$ RIOR to the advent of MRI, brainstem gliomas were regarded as a single entity; however, several studies investigating MRI have recognized that these lesions are a heterogeneous group, with certain subgroups having better prognoses for long-term survival. ${ }^{1-8}$ Essentially, brainstem gliomas are now divided into 2 categories; $20 \%$ are considered to be low grade, arising in the midbrain, medulla, or dorsal pons, and are often focal, dorsally exophytic, and amenable to resection with a relatively good prognosis. The remaining $80 \%$ of tumors arise and occupy the majority of the pons and are diffuse in nature with poor prognosis. This latter group, termed diffuse intrinsic brainstem glioma, remains one of the most challenging tumor types, with multiple clinical trials failing to improve survival. ${ }^{7,9,11,12,15}$

The aim of this study was a retrospective analysis to determine whether histological grade, age, and MRI findings are prognostic factors for survival of patients with brainstem gliomas confirmed by histopathological diagnosis.

\section{Methods}

The study involved a retrospective evaluation of medical records of 142 patients with brainstem lesions who underwent stereotactic biopsy between February 1984 and August 2007 at the Roger Salengro Hospital (Lille, France). Institutional review board approval was obtained for this study.

The procedures were performed under general anesthesia; 109 patients underwent stereotactic biopsy procedures using a Talairach halo, and 33 underwent the procedures using the robotic system (neuromate systems, Renishaw plc). Target location was determined using MRI in all cases. The target was the center of the lesion and/or area of contrast enhancement. The transfrontal approach 
was used in 123 patients, and the suboccipital transcerebellar approach was used in the remaining 19. All biopsy specimens were formalin-fixed and analyzed after staining with $\mathrm{H} \& \mathrm{E}$, Masson trichrome, and immunostains. The procedure was considered successful in cases in which a definitive diagnosis was obtained and confirmed by the clinical course of the tumor.

The overall diagnosis rate of the biopsy procedure was $93.7 \%$, and in 9 patients the biopsy results were negative. Diffuse brainstem glioma was diagnosed in 100 patients. Other neoplastic diseases were diagnosed in 23 patients. Moreover, histological evaluation revealed nonneoplastic lesions in 10 patients (Table 1). The tumors were graded according to WHO classification; low-grade gliomas were classified as Grade II and high-grade gliomas as Grades III and IV. ${ }^{10}$

Of the patients with diffuse brainstem glioma, 63 were adults, including 40 men and 23 women who ranged in age from 18 to 75 years (mean 41 years). Thirty-seven patients were children, including 19 boys and 18 girls who ranged in age from 2 to 12 years (mean 6.9 years). Patient follow-up occurred from 4 days to 278 months after the biopsy (mean 40.4 months).

Eighty-four patients received conventional focal radiotherapy; 9 patients were not treated because they presented with minor symptoms, nonprogressive tumors, and a histological diagnosis of low-grade glioma; and 7 patients died before radiotherapy was performed. Chemotherapy was administered to 40 patients at the time of relapse or when radiotherapy failed.

Regarding complications in the current series, 1 death occurred due to the procedure and 13 patients (9.8\%) presented with definitive complications.

\section{Statistical Analysis}

Data analysis was performed using Epi info (version 6.02, Centers for Disease Control) and MedCalc (version 9.3.0.9, bvba). Univariate analysis of the following variables was performed: characteristics of T1-weighted MRI studies after infusion of Gd, histological grade, and patient age.

Survival time was measured from the time of the biopsy procedure to the date of the patient's last follow-up or death due to diffuse glioma. Survival was estimated using the Kaplan-Meier method with $95 \%$ confidence intervals. Comparison of Kaplan-Meier curves between histological grades (low-grade vs high-grade glioma), characteristics of T1-weighted MRI after infusion of Gd (enhancing vs nonenhancing tumors), and age (adults vs children) were performed using the log-rank test. The Cox proportional hazards model was used to test prognostic factors in multivariate analysis. Parameters were considered statistically significant at a value of $\mathrm{p}<0.05$.

\section{Results}

The histological grade of the diffuse high-grade gliomas was determined in 51 patients $(51 \%$; 32 adults and 19 children). Diffuse low-grade gliomas were diagnosed in 49 patients (31 adults and 18 children). At the time of the last follow-up in August 2007, 55 patients (55\%) had died.
TABLE 1: Histological diagnosis according to the WHO classification of brainstem tumors in 142 patients who underwent biopsy procedures

\begin{tabular}{lc}
\hline \multicolumn{1}{c}{ Histology } & No. of Patients (\%) \\
\hline high-grade glioma & $51(36)$ \\
low-grade glioma & $49(34.6)$ \\
lymphoma & $7(4.9)$ \\
pilocytic astrocytoma & $6(4.2)$ \\
metastasis & $6(4.2)$ \\
inflammatory disease & $5(3.5)$ \\
ischemic lesion & $2(1.4)$ \\
fungal abscess & $2(1.4)$ \\
ganglioglioma & $2(1.4)$ \\
craniopharyngioma & $1(0.7)$ \\
gliosis & $1(0.7)$ \\
ependymoma & $1(0.7)$ \\
inconclusive & $9(6.3)$ \\
total & $142(100)$ \\
\hline
\end{tabular}

The mean overall survival of this population, measured from the date of biopsy, was 57 months for diffuse low-grade glioma and 13.8 months for diffuse high-grade glioma ( $\mathrm{p}<0.001$ ). A comparison between the KaplanMeier survival curves for adults and children revealed a similar median survival period of 25 and 16 months, respectively $(\mathrm{p}>0.05)$. The 1-year actuarial survival for adults was $64 \% \pm 0.6 \%$ and for children it was $66 \% \pm$ $0.08 \%$ (p > 0.05) (Fig. 1).

Figure 2 shows the comparison of Kaplan-Meier survival of the 2 subgroups of diffuse glioma. The 1-year actuarial survival rates for patients classified with lowgrade and high-grade gliomas were $87.6 \% \pm 0.04 \%$ and $34.9 \% \pm 0.8 \%$, respectively (Fig. 3 ).

In the patients with low-grade glioma, the 1-year survival rates were $92.9 \%$ for adults and $79.4 \%$ for children $(p=0.07)$. In the high-grade glioma group, the 1-year survival rates were $29.1 \%$ for adults and $44.9 \%$ for children $(p=0.34)$. These differences were not statically significant.

In the group of adults, the comparison of KaplanMeier curve survival showed higher survival in patients with low-grade glioma. The 1-year actuarial survival rates for adult patients were $92.9 \%$ for low-grade gliomas and $29.1 \%$ for high-grade gliomas. This difference was statistically significant ( $p=0.0001$ ) (Fig. 4). However, in the group of children, the 1-year actuarial survival rates for patients classified as harboring low- and high-grade gliomas were $80.4 \% \pm 0.08 \%$ and $48.6 \% \pm 0.14 \%$, respectively. This difference was not statistically significant $(\mathrm{p}$ $=0.0755)($ Fig. 5).

Regarding MRI characteristics, 51 patients (29 adults and 22 children) presented with contrast-enhancing lesions. Nonenhancing lesions were observed in 48 patients (34 adults and 14 children). One patient had a T1-weighted image without Gd infusion.

The mean survival among patients with nonenhancing lesions on MRI was 54.2 months, whereas that for pa- 


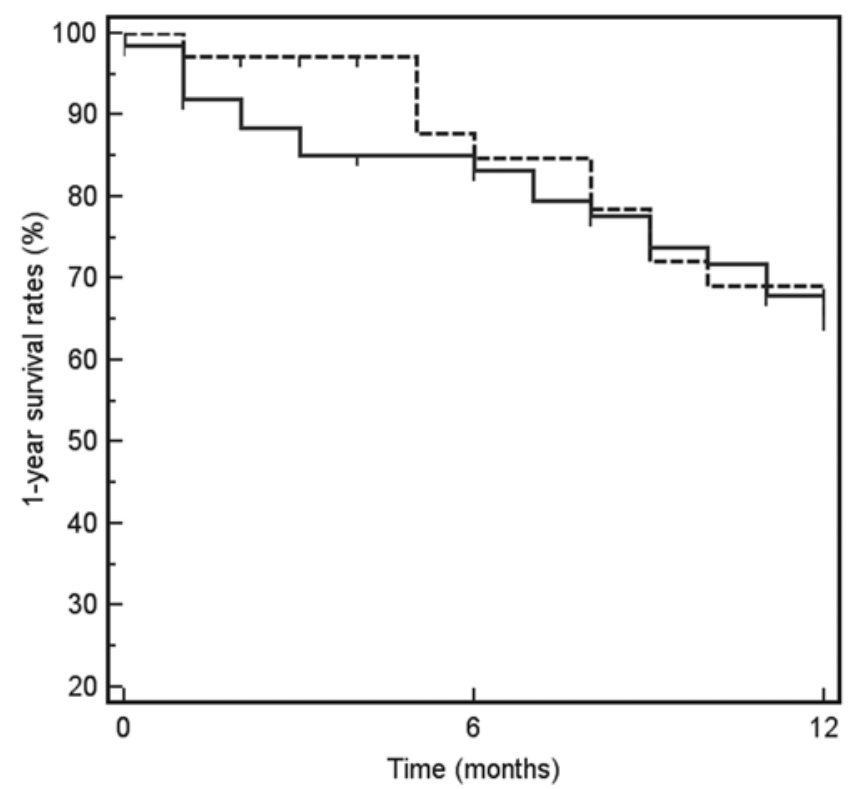

Fig. 1. Kaplan-Meier curves of the 2 subgroups of age. The solid line represents adults, while the dotted line represents children. $p>0.05$, log-rank test.

tients with enhancing lesions was 21.7 months $(\mathrm{p}<0.001)$. The Kaplan-Meier curve also revealed greater survival for patients with nonenhancing tumors (Fig. 6); moreover, the 1-year actuarial survival rate for patients with nonenhancing tumors was $88.6 \% \pm 0.04 \%$, while for enhancing tumors, it was $44.9 \% \pm 0.07 \%$ (Fig. 7).

Multivariate analysis (Cox proportional hazards regression) revealed that only histological grade $(\mathrm{p}=0.001)$ was a significant prognostic factor. The variables MRI findings $(p=0.053)$ and age $(p=0.27)$ had no statistically significant relation to patient survival in this analysis.

\section{Discussion}

In most studies, treatment decisions for brainstem glioma are based on MRI features alone and do not include histopathological diagnosis. Most authors regard biopsy procedures for intrinsic brainstem tumors as being too dangerous and consider imaging methods as sufficiently reliable. ${ }^{1,5}$ Thus, the impact of MRI findings on treatment decisions for brainstem tumors is very high, but the accuracy of MRI-based diagnosis of diffuse brainstem gliomas has not been fully verified by histopathological findings. ${ }^{8}$ Thus, some authors have reported that all diffuse brainstem gliomas located above the medulla behave as high-grade astrocytomas, and histological grade is not a significant factor for the survival of these patients. $1,4,5,18,20$

Few studies have been conducted concerning prognosis and prognostic factors in brainstem gliomas. ${ }^{1}$ This may be due to the low incidence of these tumors and the fact that histopathological diagnosis is rarely confirmed.

The present study showed that of the 3 factors studied, in univariate analysis, contrast enhancement and histological grade were significant prognostic factors, but age was not. In multivariate analysis, only histological

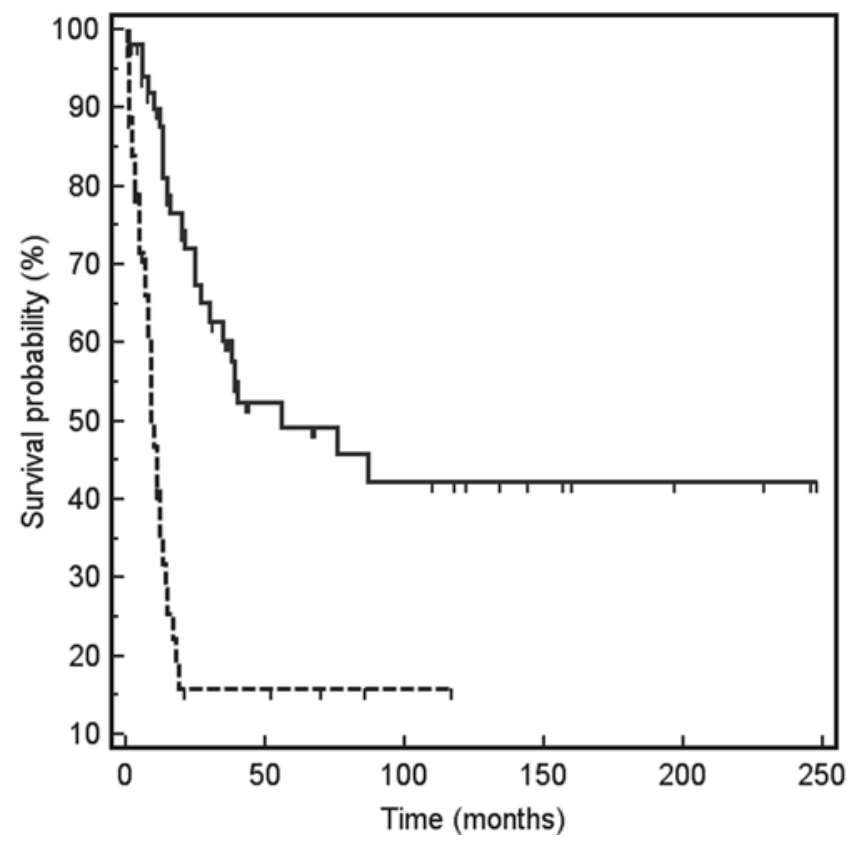

FIG. 2. Kaplan-Meier curves of the 2 subgroups of histological grade. The solid line represents diffuse low-grade glioma, while the dotted line represents diffuse high-grade glioma. $p<0.001$, log-rank test.

grade remained a significant prognostic factor. However, the histological grade was a significant prognostic factor only in the group of adults. In children, despite the longer survival of patients with low-grade glioma, the difference was not statistically significant $(\mathrm{p}=0.088)$.

These data are similar to those published by Selvapandian et al., ${ }^{17}$ who demonstrated that the tumor grade was a significant factor in predicting survival in adults,

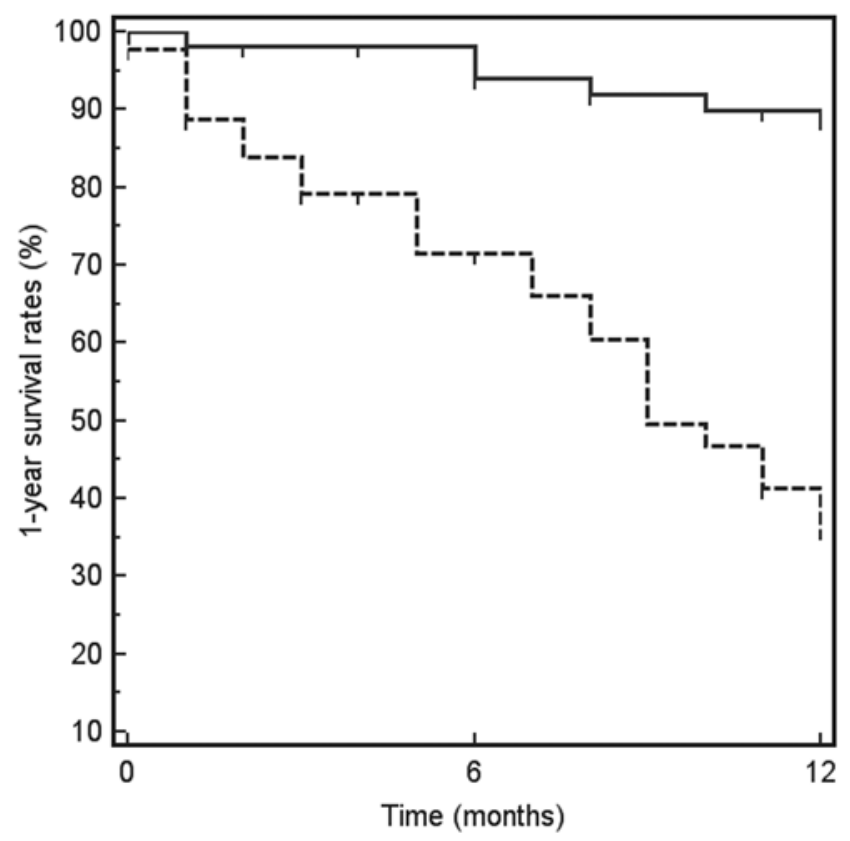

FIG. 3. Kaplan-Meier curves for 1-year actuarial survival rates. The solid line represents diffuse low-grade glioma, and the dotted line represents diffuse high-grade glioma. $p<0.001$, log-rank test. 


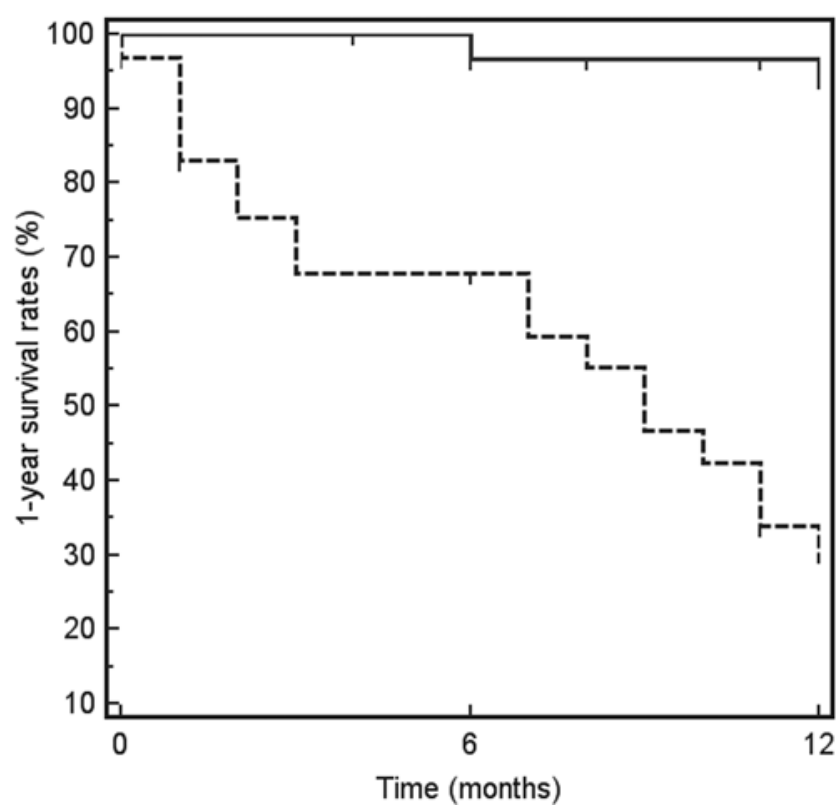

FIg. 4. Kaplan-Meier curves for 1-year actuarial survival rates in adults. The solid line represents diffuse low-grade glioma, and the dotted line represents diffuse high-grade glioma. $p=0.0001$, log-rank test.

but in children it did not correlate with outcome. However, Broniscer et al. ${ }^{3}$ showed improved outcome in children who are younger than 3 years.

Rachinger et al. ${ }^{14}$ recently reported that adult patients with diffuse low-grade gliomas showed a 1-year survival rate of $93 \%$, while this rate was $42 \%$ in patients with diffuse high-grade gliomas, also demonstrating the impact of histological grade on the survival of these patients.

Using univariate analysis, Guillamo et al. ${ }^{6}$ showed

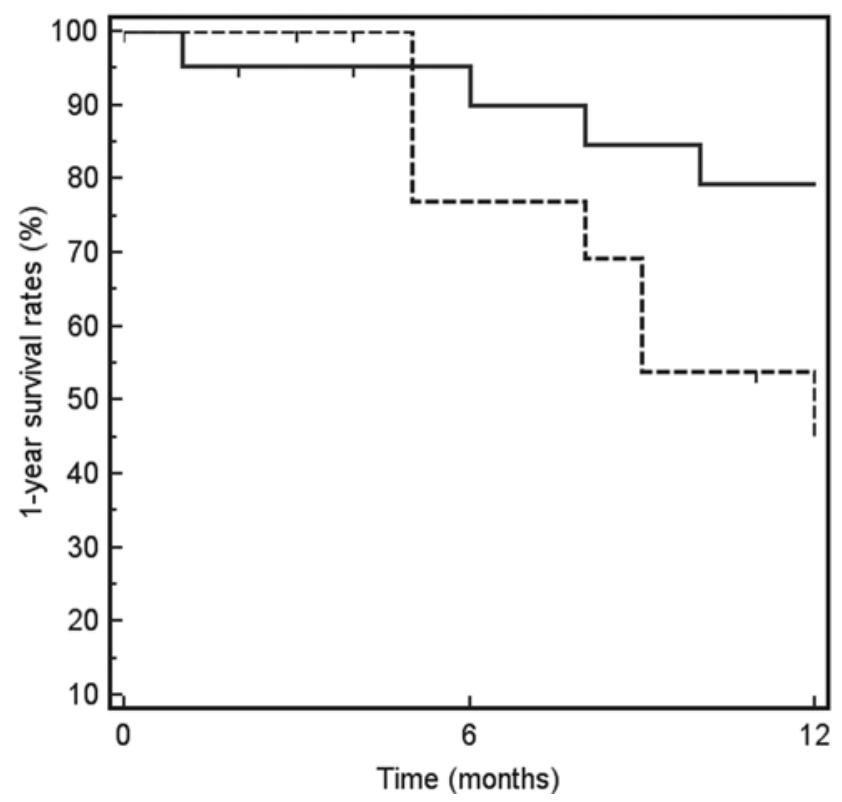

FIG. 5. Kaplan-Meier curves for 1-year actuarial survival rates in children. The solid line represents diffuse low-grade glioma, and the dotted line represents diffuse high-grade glioma. $p>0.05$, log-rank test.

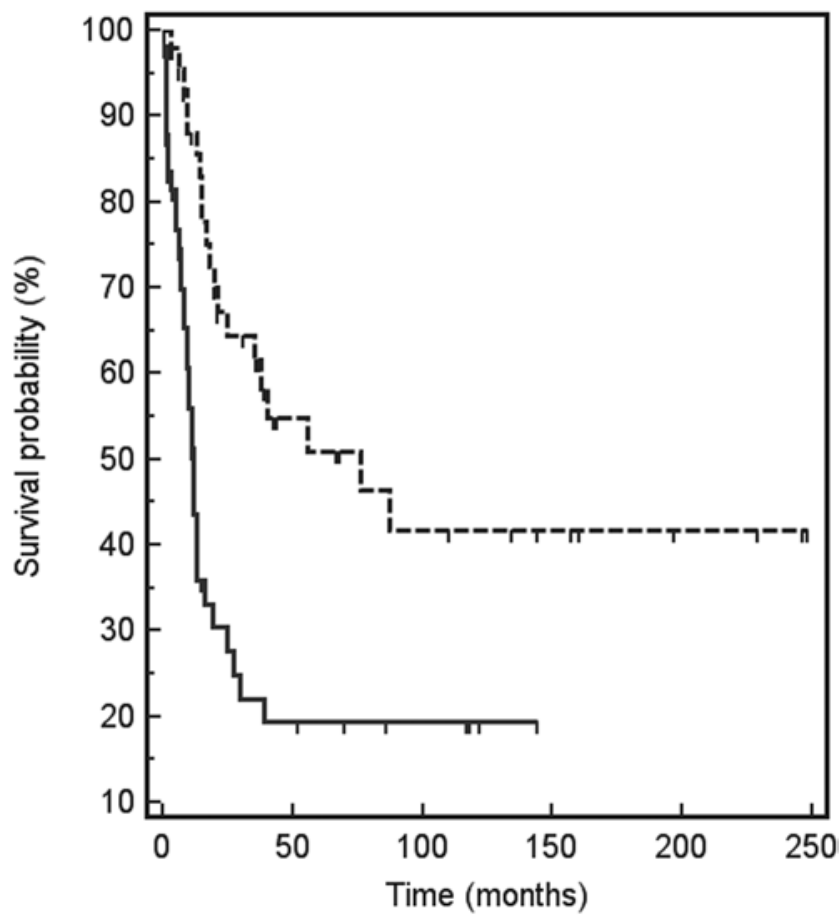

FIG. 6. Kaplan-Meier curves of the 2 subgroups of T1-weighted MRI after infusion with $\mathrm{Gd}$. The solid line represents the contrast-enhancing lesion, while the dotted line represents the nonenhancing contrast lesion. $p<0.001$, log-rank test.

in adults with diffuse glioma that histological grade and MRI "necrosis" were significant prognostic factors but that contrast enhancement not. When histological grade and MRI necrosis were included in multivariate analysis, the relative risks of these factors were similar, but only MRI necrosis was considered significant.

It is highly probable that conventional fractionated radiotherapy will not remain the only efficient treatment in diffuse brainstem glioma over the next few decades. In addition, for patients with low-grade gliomas, an initial observational policy is being adopted, followed by treatment when the patient's disease progresses clinically. ${ }^{17}$

Indeed, new chemotherapies, gene therapies, or immunotherapies, alone or in combination, will certainly succeed in improving the outcome of these patients. ${ }^{6,16,19}$ These therapies will undoubtedly require tissue sampling for diagnostic confirmation and histological grade, for molecular marker studies, or for immunological purposes prior to adopting target therapies. ${ }^{16}$ Therefore, Pollack et al. ${ }^{13}$ found that overexpression of p53 in malignant gliomas during childhood is strongly associated with adverse outcome, independent of clinical prognostic and histological findings.

\section{Conclusions}

This study revealed that histological grade and MRI features were significant prognostic factors for survival in these patients, but in multivariate analysis only histological grade remained a significant factor. Further studies should be conducted to determine whether histological 


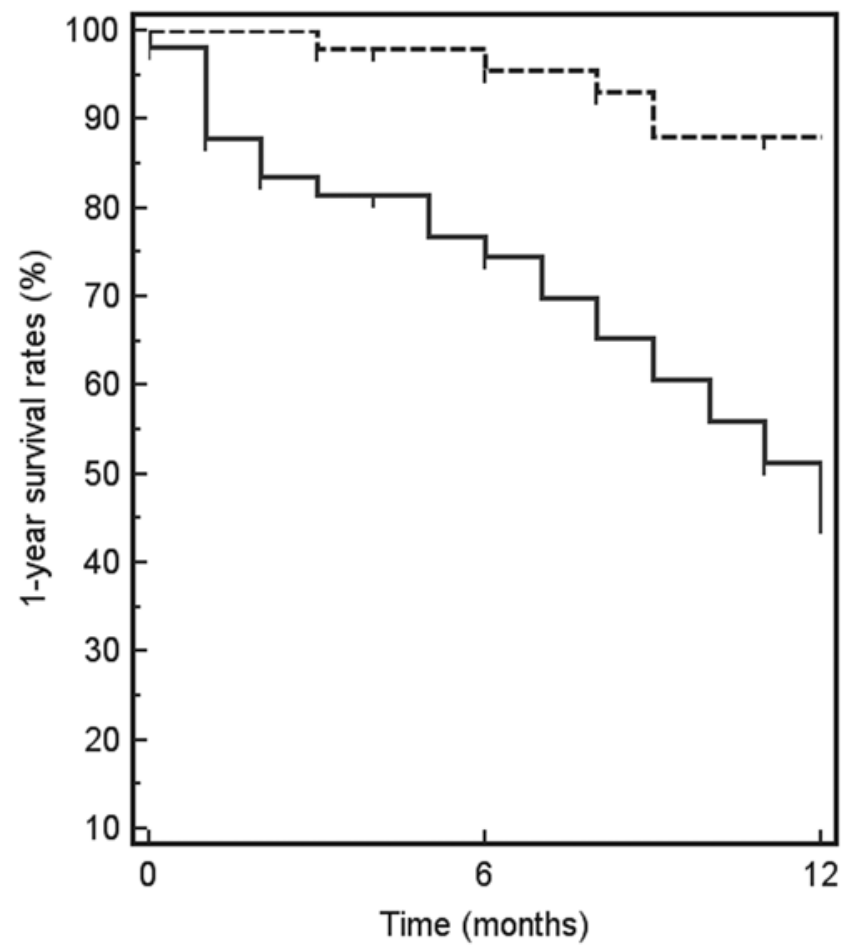

Fıg. 7. Kaplan-Meier curves for 1-year actuarial survival rates of T1-weighted MRI after infusion of Gd. The solid line represents the contrast-enhancing lesion, and the dotted line represents the nonenhancing contrast lesion. $p<0.001$, log-rank test.

grade has a greater impact than MRI alone on the treatment of these patients.

\section{Disclosure}

The authors report no conflict of interest concerning the materials or methods used in this study or the findings specified in this paper.

Author contributions to the study and manuscript preparation include the following. Conception and design: Dellaretti. Acquisition of data: Dellaretti, Dubois. Analysis and interpretation of data: Dellaretti, Reyns, Touzet, Dubois. Drafting the article: Dellaretti, Pereira. Critically revising the article: all authors. Reviewed submitted version of manuscript: all authors. Approved the final version of the manuscript on behalf of all authors: Dellaretti. Study supervision: Gusmão, Blond.

\section{References}

1. Albright AL: Diffuse brainstem tumors: when is a biopsy necessary? Pediatr Neurosurg 24:252-255, 1996

2. Behnke J, Christen HJ, Brück W, Markakis E: Intra-axial endophytic tumors in the pons and/or medulla oblongata. I. Symptoms, neuroradiological findings, and histopathology in 30 children. Childs Nerv Syst 13:122-134, 1997

3. Broniscer A, Laningham FH, Sanders RP, Kun LE, Ellison DW, Gajjar A: Young age may predict a better outcome for children with diffuse pontine glioma. Cancer 113:566-572, 2008

4. Choux M, Lena G, Do L: Brainstem tumors, in Choux M, Di
Rocco C, Hockley A, et al (eds): Pediatric Neurosurgery. London: Churchill Livingstone, 1999, pp 471-493

5. Epstein FJ, Farmer JP: Brain-stem glioma growth patterns. J Neurosurg 78:408-412, 1993

6. Guillamo JS, Monjour A, Taillandier L, Devaux B, Varlet P, Haie-Meder C, et al: Brainstem gliomas in adults: prognostic factors and classification. Brain 124:2528-2539, 2001

7. Hargrave D, Bartels U, Bouffet E: Diffuse brainstem glioma in children: critical review of clinical trials. Lancet Oncol 7: 241-248, 2006

8. Hargrave D, Chuang N, Bouffet E: Conventional MRI cannot predict survival in childhood diffuse intrinsic pontine glioma. J Neurooncol 86:313-319, 2008

9. Laigle-Donadey F, Doz F, Delattre JY: Brainstem gliomas in children and adults. Curr Opin Oncol 20:662-667, 2008

10. Louis DN, Ohgaki H, Wiestler OD, Cavenee WK: WHO Classification of Tumours of the Nervous System, ed 4. Lyon: IARC Press, 2007

11. Pincus DW, Richter EO, Yachnis AT, Bennett J, Bhatti MT, Smith A: Brainstem stereotactic biopsy sampling in children. J Neurosurg (2 Suppl) 104:108-114, 2006

12. Pirotte BJ, Lubansu A, Massager N, Wikler D, Goldman S, Levivier M: Results of positron emission tomography guidance and reassessment of the utility of an indications for stereotactic biopsy in children with infiltrative brainstem tumors. J Neurosurg (5 Suppl) 107:392-399, 2007

13. Pollack IF, Finkelstein SD, Woods J, Burnham J, Holmes EJ, Hamilton RL, et al: Expression of p53 and prognosis in children with malignant gliomas. N Engl J Med 346:420-427, 2002

14. Rachinger W, Grau S, Holtmannspoetter M, Herms J, Tonn LC, Kreth FW: Serial stereotatic biopsy of brainstem lesions in adults improves diagnostic accuracy compared with MRI only. J Neurol Neurosurg Psychiatry 80:1134-1139, 2009

15. Ranjan A, Rajshekhar V, Joseph T, Chandy MJ, Chandi SM: Nondiagnostic CT-guided stereotactic biopsies in a series of 407 cases: influence of CT morphology and operator experience. J Neurosurg 79:839-844, 1993

16. Roujeau T, Machado G, Garnett MR, Miquel C, Puget S, Geoerger B, et al: Stereotactic biopsy of diffuse pontine lesions in children. J Neurosurg 107 (1 Suppl):1-4, 2007

17. Selvapandian S, Rajshekhar V, Chandy MJ: Brainstem glioma: comparative study of clinico-radiological presentation, pathology and outcome in children and adults. Acta Neurochir (Wien) 141:721-727, 1999

18. Stroink AR, Hoffman HJ, Hendrick EB, Humphreys RP, Davidson G: Transependymal benign dorsally exophytic brain stem gliomas in childhood: diagnosis and treatment recommendations. Neurosurgery 20:439-444, 1987

19. Yen CP, Sheehan J, Steiner M, Patterson G, Steiner L: Gamma knife surgery for focal brainstem gliomas. J Neurosurg 106: $8-17,2007$

20. Zimmerman RA: Neuroimaging of primary brainstem gliomas: diagnosis and course. Pediatr Neurosurg 25:45-53, 1996

Manuscript submitted December 4, 2011.

Accepted July 23, 2012.

Please include this information when citing this paper: published online August 31, 2012; DOI: 10.3171/2012.7.JNS111992.

Address correspondence to: Marcos Dellaretti, M.D., Department of Neurosurgery, Santa Casa Hospital, 111 Santa Efigênia, Belo Horizonte, Mina Gerais, Brazil 30150-221.email: mdellaretti@mac. com. 